\title{
Estado nutricional, condições socioeconômicas, ambientais e de saúde de crianças moradoras em cortiços e favela
}

\author{
Nutritional status and health, environmental \\ and socioeconomic conditions of children \\ living in tenements and shanty towns
}

Rosangela Bezerra SANTOS ${ }^{1}$

Paula Andrea MARTINS²

Ana Lydia SAWAYA ${ }^{3}$

\section{RE S U M O}

\section{Objetivo}

Comparar a freqüência da desnutrição entre crianças de 6 meses a 6 anos, moradoras em cortiços e favela, da região central de São Paulo e caracterizar as diferenças das condições socioeconômicas, ambientais e de saúde dos dois grupos.

\section{Métodos}

Realizou-se estudo transversal com 86 crianças moradoras em cortiços e 84 crianças residentes na favela e respectivas famílias. Classificou-se a desnutrição segundo os índices estatura/idade, peso/idade e peso/estatura, ponto de corte <-1 e <-2 escore-Z, comparados ao padrão National Center for Health Statistics. Os dados socioeconômicos, ambientais e de saúde foram coletados com a aplicação de um questionário durante as visitas domiciliares. Para analisar os dados utilizaram-se o teste do qui-quadrado e o teste $t$ de Student ou o teste Mann-Whitney com nível de significância 5\%.

\section{Resultados}

A proporção da desnutrição leve foi duas vezes maior nas crianças moradoras em favela em relação às residentes nos cortiços e quatro vezes maior para desnutrição moderada. Com relação às variáveis pesquisadas, as famílias moradoras em favela apresentaram piores condições quanto a: renda, despesas com alimentação, trabalho, condições de moradia e saneamento $(p<0,001)$. Quanto às condições de saúde observou-se maior ocorrência de diarréia $(p=0,003)$ e menor cobertura vacinal $(p<0,001)$ entre as crianças moradoras em favela.

\footnotetext{
${ }^{1}$ Universidade Federal de São Paulo, Programa de Pós-Graduação em Ciências Endocrinológicas. R. Botucatu, 862 - Edifício de Ciências Biomédicas, $2^{\circ}$ andar, 04023-900, São Paulo, SP, Brasil. Correspondência para/Correspondence to: R. B. SANTOS. E-mail: <lamamede@ig.com.br>.

2 Universidade Federal de São Paulo, Departamento de Ciências da Saúde. Santos, SP, Brasil.

3 Universidade Federal de São Paulo, Departamento de Fisiologia. São Paulo, SP, Brasil.
} 


\section{Conclusão}

A freqüência da desnutrição nas crianças da favela estudada foi maior do que a encontrada nos cortiços e as famílias moradoras na favela foram consideradas extremamente pobres, com condições socioeconômicas e ambientais piores do que nos cortiços, evidenciando aspectos da pobreza urbana ligados à desnutrição infantil.

Termos de indexação: Áreas de pobreza. Desnutrição. Epidemiologia nutricional. Fatores socioeconômicos. Pré-escolar.

\section{A B S T R A C T}

\section{Objective}

The objective was to compare malnutrition rates among children aged from 6 months to 6 years living in tenements and shanty towns in downtown São Paulo city and characterize the differences in the socioeconomic, environmental and health conditions of the two groups.

\section{Methods}

A cross-sectional study was done with 86 children living in tenements and 84 children living in shanty towns and their respective families. Malnutrition was classified according to their height-for-age, weight-for-age and weight-for-height indices when compared with those of the National Center for Health Statistics. Z-scores $<-1$ and $<-2$ were used as the cut-off points of malnutrition. Socioeconomic, environmental and health data were collected by administering a questionnaire during home visits. Data were analyzed by applying the chi-square, Student's t or Mann-Whitney tests. The significance level was set at 5\%.

\section{Results}

Mild malnutrition rate was two times higher in children living in shanty towns than in those living in tenements and moderate malnutrition rate was four times higher. Regarding the analyzed variables, dwellers of shanty towns had lower incomes, spent less on food and had worse jobs and living and sanitary conditions $(p<0.001)$. Diarrhea was more frequent $(p=0.003)$ and vaccination rates were lower $(p<0.001)$ among children living in shanty towns.

\section{Conclusion}

The rate of malnutrition found for children living in the studied shanty town was higher than that found for children living in tenements. Families living in shanty towns were considered extremely poor, with worse socioeconomic and environmental conditions than those living in tenements, evidencing aspects of urban poverty associated with childhood malnutrition.

Indexing terms: Poverty areas. Malnutrition. Nutritional epidemiology. Socioeconomic factors. Child preeschool.

\section{N T R O D U Ç Ã O}

O grau e a distribuição da desnutrição em uma população dependem de fatores como: situação política e econômica, nível de educação e sanitização, produção de alimentos, prática do aleitamento materno, prevalência de doenças infecciosas, existência e efetividade de programas nutricionais e disponibilidade e qualidade dos serviços de saúde ${ }^{1}$. Sendo a desnutrição infantil um problema multifatorial, sua prevalência apresenta diferenças importantes entre países, entre regiões de um mesmo país, entre populações urbanas e rurais, entre famílias vivendo em uma mesma comunidade e entre crianças de uma mesma família².

A pobreza é a principal causa ligada à desnutrição e aos seus determinantes e ameaça a infância, ao expor milhões de crianças a doenças que poderiam ser facilmente evitadas ou curadas por meio de medicamentos e de vacinas de baixo custo ${ }^{3}$.

O Brasil, nas últimas décadas, vem confirmando uma tendência de enorme desigualdade na distribuição de renda e elevados níveis de 
pobreza, excluindo parte significativa de sua população do acesso a condições mínimas de dignidade e cidadania ${ }^{4}$. Atualmente o governo federal adota como parâmetro para dimensionar a pobreza existente no País, o salário mínimo, sendo consideradas muito pobres ou indigentes, as pessoas com renda per capita domiciliar de até um quarto do salário mínimo, e pobres aquelas com renda de até meio salário mínimo domiciliar per capita. Com base neste parâmetro, segundo dados de 2003, do total de habitantes que informaram sua renda, cerca de um terço $(31,7 \%)$ é considerado pobre, 53,9 milhões de pessoas, e com relação aos muito pobres ou indigentes, a proporção é de $12,9 \%$ ou 21,9 milhões de pessoas $^{5}$. Com o empobrecimento da população, as famílias não tiveram como arcar com os aumentos das despesas de habitação e acabaram empurradas para as condições mais precárias e irregulares para permanecer na cidade ${ }^{6}$.

As favelas e outros tipos de assentamento precário constituem a expressão mais visível dos problemas habitacionais. Logo após meados do século XX, acelerou-se na cidade de São Paulo o surgimento das favelas, associado ao agravamento da situação habitacional nos anos de 1970. Tanto que, se até 1973 a porcentagem da população favelada sobre o total do Município correspondia a $1 \%$, esta parcela elevou-se a quase $8 \%$ em 1987, ou seja, em mais de $1000 \%$, enquanto a população de São Paulo cresceu $60 \%{ }^{6}$. Segundo Marques et al. ${ }^{7}$, no ano de 2000 residiam em favelas 1160590 pessoas, aproximadamente, 11\% da população do município.

Os cortiços inseridos no processo de urbanização da cidade de São Paulo desde o final do século XIX, são, até hoje, uma opção de moradia para as pessoas pobres, em especial nas áreas centrais da cidade. São várias as denominações existentes para este tipo de moradia, entre as mais usadas estão: casa de cômodo, cabeça de porco, vila, quintal, estalagem, pensão e fileira de quartos ao longo de um corredor ${ }^{8}$. O cortiço passou por várias fases ao longo da história da cidade, primeiramente representava uma alternativa de moradia para as classes trabalhadoras, em um segundo momento, ele é, em parte, substituído pela opção das casas auto-construídas em lotes próprios da periferia e a fase atual aponta para a expansão do encortiçamento na cidade ${ }^{9}$. A marca inequívoca da moradia em cortiço é a habitação coletiva, onde em um único cômodo são exercidas várias funções (dormitório, sala e cozinha), além do uso coletivo de banheiro e tanques ${ }^{9}$.

O presente estudo buscou comparar a freqüência da desnutrição entre crianças moradoras em favela e cortiços, bem como caracterizar as diferenças entre as condições socioeconômicas, ambientais e de saúde destas duas populações.

\section{M É T O D OS}

Foi realizado um estudo transversal, com crianças na faixa etária de 6 meses a 6 anos, pertencentes às famílias moradoras em cortiços e favela do Distrito Administrativo de Santa Cecília, região central de São Paulo. Este distrito foi escolhido por ser o local onde se situa a única favela existente no centro da cidade e também por concentrar um número expressivo de cortiços. Segundo mapeamento realizado em 2002, por profissionais da Unidade Básica de Saúde Dr. Humberto Pascale, neste bairro, foram identificados cerca de 303 cortiços. A favela do Moinho, segundo dados oficiais da Prefeitura do município, existe desde 1999, nos baixos do viaduto Eng. Orlando Murgel, tendo se estendido ao longo dos anos por uma área localizada entre duas linhas de trem. São 272 barracos com cerca de 1200 habitantes ${ }^{7}$.

Foram realizadas avaliações antropométricas para coleta dos dados de peso e estatura das crianças e, posteriormente, visitas domiciliares às suas famílias para coleta dos dados sócioeconômicos, ambientais e de saúde.

Inicialmente, para ter acesso às populações do estudo, foram contatadas equipes da Pastoral da Criança, uma organização comunitária de atuação nacional que acompanha gestantes e 
crianças de 0 a 6 anos pertencentes a famílias carentes, por meio de ações que mobilizem as famílias no cuidado com seus filhos. Entre estas ações está o Dia do Peso, quando, mensalmente, é realizada a pesagem das crianças ${ }^{10}$. Das equipes da Pastoral da Criança que atuam na região do estudo, uma trabalha junto às famílias moradoras na Favela do Moinho e outras duas com famílias residentes nos cortiços. Assim, foi acordada a realização das avaliações antropométricas nos locais e nas datas já existentes no cronograma das equipes para a atividade do Dia do Peso. Nas datas estabelecidas os dados antropométricos foram coletados por duas nutricionistas (uma delas a própria pesquisadora) e duas auxiliares de enfermagem, com o apoio das equipes da Pastoral. Foram incluídas no estudo todas as crianças que compareceram às avaliações antropométricas, exceto as menores de 6 meses. Segundo dados da Pastoral, no período do estudo, residiam na favela 107 crianças na faixa etária de 6 meses a 6 anos. Com relação ao número de crianças residentes nos cortiços, não existem dados precisos. Segundo recente pesquisa da Fundação Seade ${ }^{9}$ com famílias moradoras em cortiços, realizada em nove bairros do centro da cidade, inclusive parte do Distrito Santa Cecília, há uma predominância de pessoas sozinhas ou casais sem filhos. Entre as famílias pesquisadas com filhos (40\%), mais da metade tem apenas um filho.

Favela do Moinho: foram realizadas duas avaliações antropométricas nos meses de outubro e dezembro de 2003, em uma capela existente no local, obtendo-se os dados de 84 crianças.

Cortiços: foram realizadas duas avaliações antropométricas, em dois espaços distintos (Largo Santa Cecília e Igreja Coração de Jesus), nos meses de setembro e outubro de 2004, obtendo-se os dados antropométricos de 86 crianças.

O intervalo de tempo entre as avaliações antropométricas realizadas nas duas populações ocorreu devido à impossibilidade de prosseguir com o trabalho de campo (visitas domiciliares), dentro do cronograma previamente estabelecido, em função da dificuldade de acesso à favela, por questões de segurança. No entanto, não ocorreram mudanças estruturais na condição de vida das famílias durante o período, possibilitando a comparação entre as populações estudadas, sem comprometimento da qualidade dos dados.

As medidas de peso e estatura foram verificadas de acordo com as técnicas propostas por Jelliffe ${ }^{11}$. Para a mensuração do peso das crianças com até dois anos, foi utilizada balança eletrônica portátil marca Filizola modelo BP Baby com capacidade para $15 \mathrm{~kg}$ e variações de $5 \mathrm{~g}$. Acima de dois anos as crianças foram pesadas em balança eletrônica portátil marca Kratos com capacidade de $150 \mathrm{~kg}$ e precisão de $50 \mathrm{~g}$, sem sapatos e com roupas leves.

O comprimento das crianças menores de dois anos foi obtido com o uso de um infantômetro, com a criança deitada sobre uma superfície reta. Para as crianças maiores de dois anos a altura foi medida com a utilização de uma fita métrica com divisões em centímetros e subdivisões em milímetros, fixada em uma parede plana e sem rodapés e um esquadro de madeira.

A idade das crianças foi calculada a partir da subtração da data de coleta das medidas e data de nascimento obtida a partir do cartão de vacina.

Antes da mensuração de cada criança, eram anotados os dados gerais (nome, data de nascimento, sexo), nome da mãe/responsável, endereço e, nesse momento, a mãe já era informada da realização de uma visita domiciliar, posteriormente.

A análise do estado nutricional foi realizada com base nos índices antropométricos estatura/ idade (E/I), peso/idade (P/I) e peso/estatura (P/E) em valores de escore-Z (unidades de desviopadrão), comparados ao padrão de referência do National Center for Health Statistics (NCHS 2000). Para classificação da desnutrição foram utilizados os pontos de corte $<-1$ e $<-2$ escore- $Z$.

Os dados socioeconômicos e demográficos das famílias, as condições de moradia, saneamento e de saúde das crianças foram obtidos com a 
aplicação (pela pesquisadora) de um questionário previamente testado ${ }^{12}$, durante as visitas domiciliares programadas para todas as casas das crianças que participaram das avaliações antropométricas. Neste momento a mãe/responsável era informada do estado nutricional da criança e, em caso de desnutrição, a criança era referenciada ao serviço de saúde da região. Na favela, foram realizadas 44 visitas no período de março a novembro de 2004, das 84 crianças avaliadas inicialmente, as famílias de 14 crianças não foram localizadas após dois retornos ao local, obtendose um total de 70 crianças com todas as informações coletadas. Nos cortiços, foram realizadas 64 visitas domiciliares em 30 endereços distintos, no período de outubro de 2004 a janeiro de 2005, sendo que, das 86 crianças que participaram da avaliação antropométrica, 6 crianças não tiveram suas famílias localizadas, obtendo-se um total de 80 crianças com as informações completas. Dessa forma, a avaliação do estado nutricional foi realizada com 84 crianças moradoras em favela e 86 crianças residentes em cortiços e, em relação às demais variáveis pesquisadas (características socioeconômicas, ambientais e saúde das crianças), trabalhou-se com os dados de 70 e 80 crianças, respectivamente.

\section{Análise estatística}

Para a formação do banco de dados e as análises, utilizou-se o software Epi info versão 3.3. $2^{13}$. A proporção da desnutrição entre as crianças moradoras em favela e cortiços e o comportamento das variáveis categóricas socioeconômicas, habitacionais e de saúde nas duas populações estudadas foram comparados a partir da aplicação do teste do qui-quadrado, com correção de Yates (ou teste exato de Fisher, quando necessário). As variáveis numéricas contínuas foram comparadas utilizando-se o teste $t$ de Student ou o teste de Mann-Whitney, quando a variável não apresentava uma distribuição normal. Para todas as análises utilizou-se nível de significância de 5\%.

Este estudo foi realizado em conformidade com os princípios éticos contidos na Declaração de Helsinki (2000), tendo sido avaliado e aprovado pelo Comitê de Ética em Pesquisa da Universidade Federal de São Paulo. A totalidade das mães/ responsáveis entrevistada assinou o termo de consentimento livre e esclarecido.

\section{RESULTA DOS}

A proporção de crianças com índices estatura/idade e peso/idade abaixo do ponto de corte - 1 escore-Z (Tabela 1), que classifica as crianças com déficit leve, de acordo com World Health Organization $(\mathrm{WHO})^{14}$, foi significantemente diferente entre os grupos, com uma proporção de desnutrição, aproximadamente, duas vezes maior entre as crianças moradoras em favela, quando comparadas às que residem nos cortiços. As diferenças nas proporções destes mesmos índices também foram significantes, quando considerado o ponto de corte $<-2$ escoreZ (Tabela 1), desnutrição moderada (nenhuma criança foi classificada com desnutrição grave), encontrando-se uma freqüência, aproximadamente, quatro vezes maior entre as crianças moradoras em favela em comparação às que residem em cortiços.

Na comparação das condições socioeconômicas (Tabela 2) foram observadas diferenças estatisticamente significante com relação às seguintes variáveis: composição familiar, número de cômodos, número de pessoas/cômodos $(p<0,01)$.

Tabela 1. Proporção de desnutrição em crianças moradoras em favela e cortiços, segundo os índices: estatura/idade $(E / I)$, peso/idade $(P / I)$ e peso/estatura $(P / E)$, considerando os pontos de corte $<-1$ e $<-2$ escore-Z, Distrito Santa Cecília (SP), 2005.

\begin{tabular}{|c|c|c|c|c|c|c|}
\hline \multirow{2}{*}{ Escore-Z } & \multirow{2}{*}{ Índices } & \multicolumn{2}{|c|}{ Favela $(n=84)$} & \multicolumn{2}{|c|}{ Cortiços $(n=86)$} & \multirow{2}{*}{$p^{*}$} \\
\hline & & $n$ & $\%$ & $\mathrm{n}$ & $\%$ & \\
\hline \multirow[t]{3}{*}{$<-1$ a -2} & E/l & 22 & 26,2 & 13 & 15,1 & 0,048 \\
\hline & $P / I$ & 19 & 22,6 & 10 & 11,6 & 0,037 \\
\hline & $P / E$ & 15 & 17,8 & 11 & 13,1 & 0,437 \\
\hline \multirow[t]{3}{*}{$<-2$} & $E / I$ & 11 & 13,1 & 3 & 3,5 & 0,021 \\
\hline & $P / l$ & 13 & 15,5 & 4 & 4,7 & 0,017 \\
\hline & $\mathrm{P} / \mathrm{E}$ & 4 & 4,8 & 0 & 0,0 & 0,060 \\
\hline
\end{tabular}

* Valor de $p$ teste do qui-quadrado. 
Tabela 2. Comparação entre as condições socioeconômicas das famílias moradoras em favela e cortiços, Distrito Santa Cecília (SP), 2005.

\begin{tabular}{|c|c|c|c|}
\hline Variáveis* & Favela $(n=44)$ & Cortiços $(n=64)$ & $p^{\dagger}$ \\
\hline Números de pessoas na família & $5(2-8)$ & $3(2-7)$ & $<0,001$ \\
\hline Números de filho & $3(1-6)$ & $1(1-5)$ & $<0,001$ \\
\hline Número de cômodos & $2(1-5)$ & $1(1-2)$ & $<0,001$ \\
\hline Número pessoas/cômodos & $2(0,7-6)$ & $3(1,5-7)$ & $<0,001$ \\
\hline Escolaridade materna (anos de estudo) $)^{\ddagger}$ & $2(0-11)$ & $4(0-11)$ & 0,142 \\
\hline Escolaridade do pai/companheiro (anos de estudo)s & $3,5(0-11)$ & $5,0(0-15)$ & 0,060 \\
\hline Renda per capita $(\mathrm{R} \$)$ & $37,75(0,00-157,40)$ & $166,67(33,33-537,50)$ & $<0,001$ \\
\hline Despesas mensais aluguel $(R \$)^{\prime \prime}$ & $75,00(50,00-100,00)$ & $250,00(65,00-900,00)$ & 0,002 \\
\hline Água, luz, gás $(R \$)^{\Uparrow}$ & $32,00(30,00-35,00)$ & $32,00(30,00-420,00)$ & 0,039 \\
\hline Alimentação $(R \$) * *$ & $70,00(15,00-250,00)$ & $200,00(20,00-400,00)$ & $<0,001$ \\
\hline
\end{tabular}

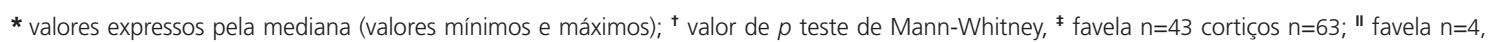
cortiços $n=54$; ** favela: $n=43$, cortiços $n=62 ;{ }^{\S}$ favela $n=32$, cortiços $n=49$; ^ favela $n=32$, despesa referente somente a gás; cortiços $n=42$.

Tabela 3. Comparação entre as condições ambientais e o vínculo empregatício, das famílias moradoras em favela e cortiços, Distrito Santa Cecília (SP), 2005.

\begin{tabular}{|c|c|c|c|c|c|}
\hline \multirow{2}{*}{ Variáveis } & \multicolumn{2}{|c|}{ Favela $(n=44)$} & \multicolumn{2}{|c|}{ Cortiços $(n=64)$} & \multirow{2}{*}{$p^{*}$} \\
\hline & $\mathrm{n}$ & $\%$ & $\mathrm{n}$ & $\%$ & \\
\hline Tipo de construção & & & & & $<0,001$ \\
\hline Madeira & 39 & 88,6 & 0 & 0,0 & \\
\hline Mista/Alvenaria & 5 & 11,4 & 64 & 100,0 & \\
\hline Revestimento do piso $^{\dagger}$ & 12 & 27,3 & 55 & 85,9 & $<0,001$ \\
\hline Refrigerador no domicílio $^{\dagger}$ & 29 & 65,9 & 59 & 92,2 & 0,001 \\
\hline \multicolumn{6}{|l|}{ Destino dos dejetos } \\
\hline Esgoto & 3 & 6,8 & 58 & 90,6 & $<0,001$ \\
\hline Fossa/céu aberto & 41 & 93,2 & 6 & 9,4 & \\
\hline \multicolumn{6}{|l|}{ Uso do WC } \\
\hline Unifamiliar & 44 & 100,0 & 4 & 6,2 & $<0,001$ \\
\hline Coletivo & 0 & 0,0 & 60 & 93,8 & \\
\hline \multicolumn{6}{|l|}{ Destino lixo } \\
\hline Coleta pública & 0 & 0,0 & 64 & 100,0 & $<0,001$ \\
\hline Céu aberto & 44 & 100,0 & 0 & 0,0 & \\
\hline \multicolumn{6}{|l|}{ Abastecimento água } \\
\hline Domiciliar & 12 & 27,3 & 22 & 34,4 & 0,568 \\
\hline Coletivo & 32 & 72,7 & 42 & 65,6 & \\
\hline Falta de água ${ }^{\dagger}$ & 14 & 31,8 & 11 & 17,2 & \\
\hline \multicolumn{6}{|l|}{ Tratamento da água } \\
\hline Nenhum & 17 & 38,6 & 18 & 28,6 & 0,123 \\
\hline Fervura, filtração, cloração & 27 & 61,4 & 46 & 71,4 & 0,377 \\
\hline \multicolumn{6}{|l|}{ Mães com vínculo } \\
\hline empregatício (registro em carteira) ${ }^{t, \neq}$ & 4 & 9,5 & 15 & 23,8 & 0,046 \\
\hline Pais/companheiros com vínculo & 5 & 156 & 29 & 59.2 & $<0001$ \\
\hline
\end{tabular}

* Valor de $p$ teste do qui-quadrado; ${ }^{\dagger}$ Variáveis dicotômicas - valores apresentados referem-se às respostas afirmativas; ${ }^{*}$ favela $n=43$, cortiços $n=63 ; \Im$ favela $n=32$, cortiços $n=49$. 
A escolaridade dos responsáveis pelas famílias apresenta-se baixa nas duas populações do estudo, variando de 2 a 5 anos de estudo.

As pessoas que residem na favela apresentam uma renda per capita mediana que corresponde a, aproximadamente, $20 \%$ da renda per capita das pessoas moradoras nos cortiços $(p<0,001)$.

Com relação aos gastos com alimentação, observa-se que as famílias moradoras na favela (constituídas por um maior número de pessoas) apresentam uma despesa com alimentação três vezes menor, em relação aos gastos com alimentos das famílias moradoras em cortiços.

Os dados sobre as condições de moradia e saneamento (Tabela 3) mostram diferenças estatisticamente significantes entre os dois grupos estudados, quanto a: tipo de construção, revestimento no piso, presença de refrigerador no domicílio, utilização da rede de esgotos, uso do banheiro (unifamiliar ou coletivo), coleta de lixo. Quanto ao vínculo empregatício dos responsáveis pelas famílias, observa-se que a maioria das mães e pais/companheiros moradores na favela não possui registro em carteira, enquanto nos cortiços, mais da metade dos pais/companheiros são registrados.

Na Tabela 4 observa-se diferença estatisticamente significante com relação à ocorrência de diarréia, que foi observada em 37\% das crianças moradoras em favela e em $15 \%$ das crianças residentes em cortiços. A cobertura vacinal das crianças que moram nos cortiços foi quase total, enquanto na favela $23 \%$ das crianças estavam com alguma vacina atrasada.

Os dados antropométricos e as condições de aleitamento das crianças estudadas (Tabela 5) mostram, com relação ao estado nutricional, que os índices estatura/idade e peso/idade apresentam

Tabela 4. Comparação entre as condições de saúde das crianças moradoras em favela e cortiços, Distrito Santa Cecília (SP), 2005.

\begin{tabular}{|c|c|c|c|c|c|}
\hline & \multicolumn{2}{|c|}{ Favela $n=70$} & \multicolumn{2}{|c|}{ Cortiços $n=80$} & \multirow{2}{*}{$p^{*}$} \\
\hline & $\mathrm{n}$ & $\%$ & $\mathrm{n}$ & $\%$ & \\
\hline \multicolumn{6}{|l|}{$N^{\circ}$ consulta pré-natal ${ }^{t}$} \\
\hline$\geq 6$ & 51 & 72,9 & 60 & 76,8 & 0,703 \\
\hline$<6$ & 19 & 27,1 & 18 & 23,1 & \\
\hline \multicolumn{6}{|l|}{$\operatorname{SexO}^{\ddagger}$} \\
\hline Masculino & 37 & 44,0 & 45 & 52,3 & 0,354 \\
\hline Feminino & 47 & 56,0 & 41 & 47,7 & \\
\hline Diarréia§ & 26 & 37,1 & 12 & 15,0 & 0,003 \\
\hline Problemas respiratórios $§$ & 41 & 58,6 & 35 & 43,8 & 0,090 \\
\hline Internação§ & 34 & 48,6 & 26 & 32,5 & 0,060 \\
\hline Vacinas em dia§ & 54 & 77,1 & 77 & 96,3 & $<0,001$ \\
\hline $\begin{array}{l}\text { Matrícula em posto de } \\
\text { saúde }^{\S}\end{array}$ & 65 & 92,9 & 73 & 91,3 & 0,951 \\
\hline
\end{tabular}

* Valor de $p$ teste do qui-quadrado; ${ }^{\dagger}$ Cortiços - 2 crianças sem informação; ${ }^{\ddagger}$ Favela: $n=84$, Cortiços: $n=86$; ${ }^{\S}$ Variáveis dicotômicas - valores apresentados referem-se às respostas afirmativas.

Tabela 5. Comparação entre as variáveis antropométricas e as condições de aleitamento das crianças moradoras em favela e cortiços, Distrito Santa Cecília (SP), 2005.

\begin{tabular}{|c|c|c|c|c|c|}
\hline \multirow{2}{*}{ Variáveis* } & \multicolumn{2}{|c|}{ Favela $(n=84)$} & \multicolumn{2}{|c|}{ Cortiços $(n=86)$} & \multirow{2}{*}{$p^{\dagger}$} \\
\hline & M & DP & M & DP & \\
\hline Idade (anos) & 2,00 & $(0-6,00)^{\pi}$ & 3,00 & $(0-6,00)^{\pi}$ & $0,276^{* *}$ \\
\hline Estatura $(\mathrm{cm})$ & 89,49 & 16,12 & 94,54 & 13,17 & 0,027 \\
\hline Peso (kg) & 13,23 & 4,49 & 14,80 & 3,83 & 0,015 \\
\hline Peso de nascimento $(\mathrm{g})^{\ddagger}$ & 32,50 & 579,94 & 3131,00 & 532,39 & 0,212 \\
\hline Estatura/Idade (escore-Z) & $-0,87$ & 1,05 & $-0,29$ & 1,01 & $<0,001$ \\
\hline Peso/ldade (escore-Z) & $-0,61$ & 1,02 & $-0,06$ & 1,06 & $<0,001$ \\
\hline Peso/Estatura (escore-Z) & $-0,03$ & 0,92 & 0,22 & 0,96 & 0,070 \\
\hline Introdução da mamadeira (meses)§ & 2,00 & $(0,2-25,00)^{n}$ & 5,00 & $(0,25-28,00)^{\pi}$ & $0,016^{* *}$ \\
\hline Interrupção do aleitamento materno (meses)" & 6,50 & $(0,2-48,00)^{n}$ & 7,00 & $(0,25-42,00)^{\pi}$ & $0,514^{* *}$ \\
\hline
\end{tabular}

* Valores expressos em média (M) e desvio-padrão (DP); ** Valor de $p$ teste de Mann-Whitney; favela: $\mathrm{n}=56$, cortiços $\mathrm{n}=677^{\dagger}$ Valor de $p$ teste t- Student; " Mediana, valores mínimos e máximos; ${ }^{\ddagger}$ favela: $n=62$; cortiços $n=74$; ${ }^{\S}$ favela: $n=52$; cortiços $n=59$, " favela: $n=56$, cortiços $n=67$. 
diferenças significantes na comparação entre as populações estudadas $(p<0,001)$, com valores menores na população moradora em favela.

A introdução da mamadeira antes dos seis meses mostra uma diferença significante entre as duas populações do estudo, com idade mediana de 2 meses para as crianças moradoras na favela e de 5 meses para as residentes nos cortiços $(p=0,016)$.

\section{I S C U S S Ã O}

Apesar da melhoria das condições nutricionais das crianças no Brasil, a desnutrição tem ocorrido de forma muito desigual e o problema ainda é muito grave nas regiões mais pobres do País e nos bolsões de pobreza das grandes cidades $^{15}$.

O ponto de corte recomendado internacionalmente para classificar a criança como desnutrida (escore-Z abaixo de 2 desvios-padrão, comparado ao valor médio da referência internacional ${ }^{16}$ tem sido utilizado nas pesquisas populacionais, identificando as prevalências e as tendências da desnutrição moderada e grave. Comparando-se os resultados deste estudo à última pesquisa populacional realizada no Brasil, que avaliou a prevalência da desnutrição em crianças menores de 5 anos, PNDS, 1996, constata-se que a freqüência de desnutrição nas crianças moradoras em favela, de acordo com os três índices antropométricos, é superior à prevalência encontrada em 1996 no Brasil e em São Paulo ${ }^{15}$, mesmo considerando-se a diferença nos padrões de referência utilizados, no presente estudo (NCHS 2000) e na referida pesquisa (NCHS 1977). O resultado desta comparação assemelha-se aos dados obtidos em estudo realizado por Domene et al. ${ }^{17}$, que avaliou a prevalência da desnutrição infantil, nos bolsões de pobreza do município de Campinas, SP.

Com relação à freqüência de desnutrição moderada nas crianças moradoras em cortiços, esta se mostrou abaixo dos índices encontrados no estudo populacional nacional de $1996^{15}$ e apresentou o mesmo déficit de peso/idade encontrado em São Paulo.

Quando a desnutrição é analisada segundo o ponto de corte <-1 escore-Z, a freqüência apresenta-se alta nas duas populações do estudo. A desnutrição classificada como déficit leve, embora pouco utilizada em estudos populacionais, tem a importância de sua avaliação reconhecida em estudos que demonstram sua associação aos níveis de mortalidade infantil e às alterações metabólicas já identificadas neste grau de desnutrição ${ }^{18,19}$.

Frente à natureza multicausal da desnutrição, muitos estudos têm buscado evidenciar os efeitos das condições socioeconômicas, ambientais, demográficas, biológicas, entre outras, sobre o estado nutricional ${ }^{2,19-21}$. No presente estudo, observa-se que, apesar da vulnerabilidade a que todas as famílias estão expostas, as condições de precariedade entre as duas populações são distintas.

Segundo Marques \& Torres $^{22}$, as favelas da região central são bastante peculiares em termos urbanos, observa-se maior precariedade de serviços urbanos, piores taxas de escolaridade e salários médios mais baixos, o que foi evidenciado neste estudo quanto às famílias moradoras na favela.

Nos cortiços, a precariedade das condições de moradia é caracterizada pela ocupação de um único cômodo pelas famílias e o uso coletivo do banheiro e do tanque ${ }^{9}$. Quase a totalidade das famílias estudadas residentes em cortiços apresentou esta condição de moradia, dado também encontrado em recente pesquisa socioeconômica realizada pela Fundação Seade ${ }^{9}$, em nove bairros representativos da maior concentração de cortiços do município de São Paulo.

Durante as visitas domiciliares foi possível perceber que há diferenças significativas em relação à conservação dos imóveis onde residem as famílias encortiçadas. Nos imóveis com condições de moradia extremamente precárias - sobre- 
carga na rede elétrica com risco de incêndio, infiltrações de água, entupimento das canalizações, falta de ventilação natural nos cômodos (devido à ausência de janela ou à presença de divisórias de madeira para dividir o cômodo), baixo valor de aluguel em comparação aos imóveis bem conservados - residem as famílias estudadas que possuem um maior número de filhos, variando de dois a cinco. De acordo com Piccini ${ }^{8}$, a qualidade dos cortiços é caracterizada pela falta de manutenção do imóvel, o que foi evidenciado neste estudo.

O trabalho informal, chamado bico, que tem como característica a sua instabilidades é a principal fonte de renda da maioria dos moradores da favela, gerando uma renda per capita mensal inferior a $1 \frac{1}{4}$ do salário mínimo vigente na época do estudo ( $R \$ 260,00)$, valor que classifica estas pessoas como extremamente pobres ${ }^{2}$. Apenas uma pequena parcela dos responsáveis tem vínculo empregatício com registro em carteira. Um estudo realizado por Ferrari23 em uma favela da região sudoeste da cidade, que também analisou o vínculo empregatício dos responsáveis pelas famílias, apontou que metade das mães que trabalhavam era registrada e, entre os pais, 2/3 deles eram registrados, índices bem superiores aos encontrados neste estudo.

Nas famílias estudadas residentes em cortiços, observa-se que o trabalho assalariado com registro em carteira predomina como principal fonte de renda. A renda per capita mensal mediana destas famílias é ligeiramente superior à linha de pobreza adotada pelo Governo Federal que considera como pobres, as pessoas com renda per capita de até meio salário mínimo ${ }^{5}$. Os dados apresentados pela pesquisa da Fundação Seade ${ }^{9}$ quanto ao vínculo empregatício dos chefes de família são semelhantes aos encontrados neste estudo, de acordo com a pesquisa parcela expressiva dos chefes das famílias encortiçadas é assalariada com registro em carteira.

Os dados demográficos das populações estudadas (não apresentados em tabela) e a baixa escolaridade confirmam achados de literatura que demonstram a existência de uma parcela significativa de migrantes entre os mais pobres, oriunda de outras regiões, principalmente do Nordeste. A população migrante com baixos níveis de escolaridade e, portanto, com maior dificuldade de acesso aos postos de trabalho, consegue integrarse apenas precariamente ao mercado de trabalho urbano, permanecendo em grande medida desempregada ou subempregada22.

Na saúde da criança, particularmente o nível socioeconômico, o saneamento básico, o acesso aos serviços de saúde, entre outros fatores, têm sido amplamente estudados, demonstrando a associação dessas condições à morbimortalidade e à desnutrição ${ }^{24}$.

O estado de saúde ao nascer é o fator determinante de maior importância para a sobrevivência e qualidade de vida da criança $a^{25}$. No presente estudo, observa-se que o peso de nascimento das crianças, que tiveram este dado coletado, mostrou-se adequado, sugerindo que as condições de vida pós-natal destas crianças, em especial das moradoras em favela, determinam a maior proporção de desnutrição nesta comunidade.

Vários estudos têm demonstrado a associação entre a ocorrência de diarréia e o baixo poder aquisitivo das famílias, falta de disponibilidade de água domiciliar, lixo ambiental26,27, fatores que estão presentes na favela em que residem as crianças estudadas, e nestas a ocorrência de diarréia foi maior, quando comparada à observada entre as crianças residentes nos cortiços.

Com relação aos problemas respiratórios, convém ressaltar que sendo este um estudo transversal, a coleta deste dado depende da memória do informante e de quanto o episódio chamou sua atenção. No entanto, sabe-se que as intercorrências infecciosas (especialmente diarréia e infecções respiratórias) pertencem ao grupo de fatores conhecidamente associados à desnutrição e quanto maior a freqüência e a gravidade dos episódios, maior o efeito deletério sobre o estado nutricional da criança. No caso das populações estudadas, o percentual significativo de crianças 
que apresentaram problemas respiratórios pode estar relacionado às condições insalubres de moradia em que vivem.

A substituição precoce do leite materno por alimentos complementares tem constituído o eixo explicativo para o déficit precoce do crescimento linear de crianças de países subdesenvolvidos, quando comparado com o início do déficit ponderal ${ }^{28}$. Está também associada à maior ocorrência de anemia e doenças infecciosas, particularmente gastrintestinais e respiratórias ${ }^{29}$. No presente estudo, pode-se observar que embora o aleitamento materno estivesse presente, a introdução da mamadeira foi precoce nas duas populações estudadas, principalmente entre as crianças residentes na favela. Apesar da proteção conferida pelo aleitamento materno, a introdução precoce da mamadeira expõe estas crianças a maiores riscos de contaminação (condições precárias de higiene) e a erros alimentares.

\section{O N CLUS Ã O}

Este estudo sugere o fato já bem documentado de que a pobreza é a principal causa ligada à desnutrição. As famílias moradoras em favela foram consideradas extremamente pobres, com condições socioeconômicas e ambientais extremamente precárias e nestas, a proporção de desnutrição leve e moderada foi maior em relação à encontrada entre as crianças residentes nos cortiços. Destaca-se ainda que os estudos sobre cortiços são raros, por isso o presente trabalho contribui para o conhecimento de uma realidade pouco visível, devido, em parte, à sua inserção na malha urbana. Assim, as crianças pertencentes às famílias encortiçadas estão expostas às condições insalubres deste tipo de moradia, que se agravam nos imóveis deteriorados pela falta de manutenção e nestes locais as condições de precariedade se assemelham às encontradas na favela.

Frente aos achados deste estudo, pode-se delinear a continuidade do mesmo com a utilização de um modelo teórico e de técnicas de análise que permitam identificar os fatores de risco mais associados à desnutrição, nas duas populações estudadas.

\section{COLABORADORES}

R.B. SANTOS trabalhou na concepção do estudo, na coleta dos dados, na análise e na interpretação dos resultados e na redação do manuscrito. P.A. MARTINS elaborou o banco de dados, colaborou na análise estatística dos dados e na redação do manuscrito. A. L. SAWAYA contribuiu com a interpretação dos resultados, a discussão e a redação final.

\section{REFER Ê N CIAS}

1. Müller $\mathrm{O}$, Krawinkel M. Malnutrition and health in developing countries. CMAJ. 2005; 173(3): 279-86.

2. Olinto MTA, Víctora CG, Barros FC, Tomasi E. Determinantes da desnutrição infantil em população de baixa renda: um modelo de análise hierarquizado. Cad Saúde Pública. 1993; 9(Supl 1):14-27.

3. Fundação das Nações Unidas para Infância. Situação mundial da infância 2005. Infância ameaçada [acesso 200518 set]. Disponível em: $<$ <ttp://www.unicef.org.br/>.

4. Barros RP, Henriques R, Mendonça R. Desigualdade e pobreza no Brasil: retrato de uma estabilidade inaceitável. Rev Bras Ciênc Soc. 2000; 15(42): 123-42.

5. Instituto de Pesquisa Econômica Aplicada. Radar social 2005 [acesso 2005 out 12]. Disponível em: $<$ http://www.ipea.gov.br>.

6. Sampaio MRA, Pereira PCX. Habitação em São Paulo. Estud Av. 2003; 17(48):167-83.

7. Marques E, Torres H, Saraiva C. Favelas do Município de São Paulo: estimativas de população para os anos de 1991, 1996 e 2000. Base cartográfica digital das favelas do município de São Paulo [CD-ROM]. São Paulo: Prefeitura Municipal de São Paulo/Centro de Estudos da Metrópole; 2002.

8. Piccini A. Cortiços em São Paulo: conceito e preconceito na reestruturação do centro urbano de São Paulo. São Paulo: Annablume; 1999.

9. Fundação Sistema Estadual de Análises de Dados. Relatório: setores básicos de intervenção do Programa de Atuação em Cortiços. Dados cadastrais e sócio-econômicos do município de São Paulo. São Paulo: Fundação Seade; 2003 
10. Pastoral da Criança. Quem atendemos. Ações [acesso 2007 jan 15]. Disponível em: <http://www. pastoraldacrianca.org.br>.

11. Jelliffe DB. Evaluación del estado de nutrición de la comunidad. Ginebra: Organización Mundial e Salud; 1968. OMS - Série de Monografias.

12. Sawaya AL, Dallal G, Solymos G, Souza MH, Ventura $\mathrm{ML}$, Roberts $\mathrm{SB}$, et al. Obesity and malnutrition in a shantytown population in the city of São Paulo, Brazil. Obes Res. 1995; 3(Supl 2):107-15.

13. Centers for Disease Control and Prevention. Epi Info [computer program]. Version 3.3.2 [access 2005 Mar 12]. Available from: <http://www. cdc.gov/epiinfo/downloads.htm>.

14. World Health Organization. Physical status: the use and interpretation of antropometry. Geneva: WHO; 1995. Technical Report Series, 854.

15. Portal Vencendo a Desnutrição. Perfil da desnutrição caso Brasil. Prevalências e tendências 2002 [acesso 2005 dez 26]. Disponível em: <http://www. desnutricao.org.br/home.htm>.

16. Blössner M, Onis M. Malnutrition: quantifying the health impact at national and local levels. Geneve: WHO; 2005. Environmental Burden of Disease Series, $\mathrm{n}^{\circ} 12$.

17. Domene SMA, Zabotto CB, Meneguello R, Galeazzi MAM, Taddei JAAC. Perfil nutricional de crianças e suas mães em bolsões de pobreza do município de Campinas, SP-1996. Rev Nutr [periódico na Internet]. 1999 ago [acesso 2008 mar]; 12(2):18389. Disponível em: <http://www.scielo.br>. doi: 10. 1590/S1415-52731999000200008.

18. Pelletier DL. The relationship between child antropometry and mortality in developing countries: Implications for policy, programs and future research. J Nutr. 1994; 124(Supl 10): 2047-81.

19. Grillo LP, Carvalho LR, Silva AC, Verreshi ITN, Sawaya AL. Influência das condições socioeconômicas nas alterações nutricionais e na taxa de metabolismo de repouso em crianças escolares moradoras em favelas no município de São Paulo. Rev Assoc Med Bras. 2000; 46(1):7-14.

20. Engstrom EM, Anjos LA. Déficit estatural nas crianças brasileiras: relação com condições sócio- ambientais e estado nutricional materno. Cad Saúde Pública. 1999; 15(3):559-67.

21. Teixeira JC. Fatores ambientais associados à desnutrição infantil em área de invasão, Juiz de Fora, MG. Rev Bras Epidemiol. 2004; 7(3):270-8.

22. Marques $E$, Torres $H$, organizadores. São Paulo: segregação, pobreza e desigualdades sociais. São Paulo: Senac; 2005.

23. Ferrari AA. Fatores de risco para desnutrição energético-protéica em pré-escolares de uma favela de São Paulo [dissertação]. São Paulo: Universidade Federal de São Paulo; 1996.

24. Puccini RF, Wechster R, Silva EMK, Resegue R. Fatores de risco para morbidade e desnutrição em crianças acompanhadas em programa de atenção à saúde da criança. J Pediatr. 1997; 73(4):244-51.

25. Luz TP, Neves LAT, Reis AFF, Silva GR, Silva LGP. Magnitude do problema do baixo peso ao nascer. J Bras Ginecol. 1998; 108(5):133-44.

26. Vanderlei LCM, Silva GAP, Braga JU. Fatores de risco para internamento por diarréia aguda em menores de dois anos: estudo de caso-controle. Cad Saúde Pública. 2003; 19(2):455-63.

27. Borges CVD, Veiga APB, Barroso GS, Jesus EFO, Serpa RFB, Moreira S, et al. Associação entre concentrações séricas de minerais, índices antropométricos e ocorrência de diarréia entre crianças de baixa renda da região metropolitana do Rio de Janeiro. Rev Nutr. 2007; 20(2):159-69.

28. Oliveira LPM, Assis AMO, Pinheiro SMC, Prado MS, Barreto ML. Alimentação complementar nos primeiros dois anos de vida. Rev Nutr [periódico na Internet]. 2005 ago [acesso 2008 nov 25];18(4):459-69. Disponível em: <http://www. scielo.br>. doi: 10.1590/S1415-52732005000 400002.

29. Oliveira LPM, Assis AMO, Gomes GSS, Prado MS, Barreto ML. Duração do aleitamento materno, regime alimentar e fatores associados segundo condições de vida em Salvador, Bahia, Brasil. Cad Saúde Pública. 2005; 21(5):1519-30.

Recebido em: 16/5/2007

Versão final reapresentada em: 23/4/2008 Aprovado em: 18/8/2008 